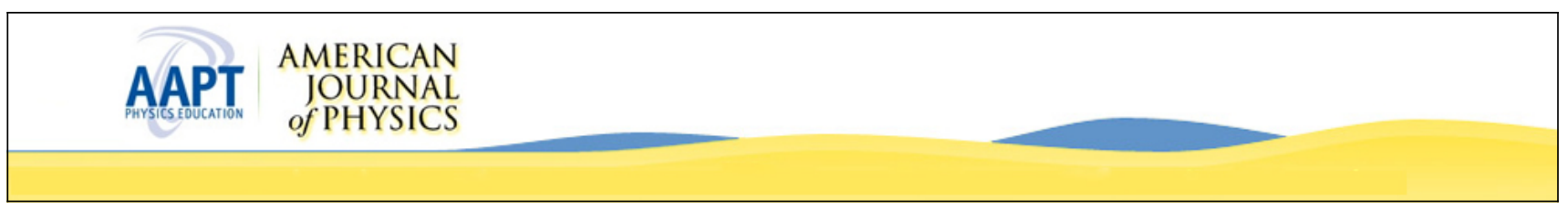

\title{
A sensitive photodiode photometer for laboratory experiments
}

David L. DuPuy

Citation: Am. J. Phys. 46, 1079 (1978); doi: 10.1119/1.11432

View online: http://dx.doi.org/10.1119/1.11432

View Table of Contents: http://ajp.aapt.org/resource/1/AJPIAS/v46/i10

Published by the American Association of Physics Teachers

\section{Related Articles}

Laboratory Investigation of Noise-Canceling Headphones Utilizing "Mr. Blockhead"

Phys. Teach. 51, 332 (2013)

The use of dc glow discharges as undergraduate educational tools

Am. J. Phys. 81, 663 (2013)

Resistivity in Play-Doh: Time and Color Variations

Phys. Teach. 51, 351 (2013)

Corrected Launch Speed for a Projectile Motion Laboratory

Phys. Teach. 51, 359 (2013)

Improving the quantification of Brownian motion

Am. J. Phys. 81, 485 (2013)

\section{Additional information on Am. J. Phys.}

Journal Homepage: http://ajp.aapt.org/

Journal Information: http://ajp.aapt.org/about/about_the_journal

Top downloads: http://ajp.aapt.org/most_downloaded

Information for Authors: http://ajp.dickinson.edu/Contributors/contGenlnfo.html

\section{ADVERTISEMENT}

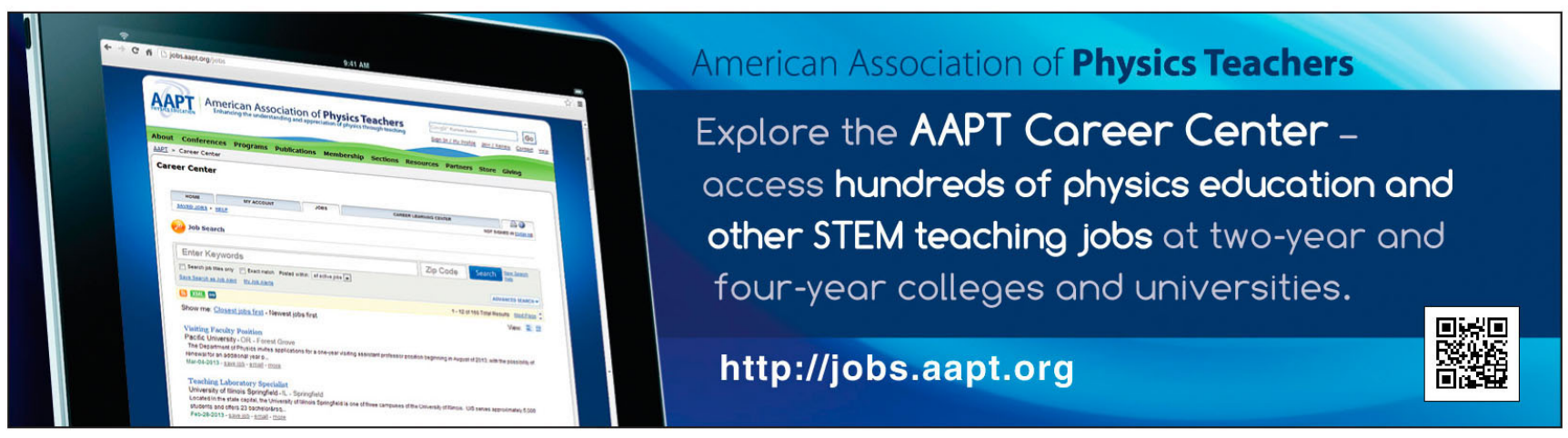




\title{
APPARATUS NOTES
}

\author{
Bruce G. Eaton, Editor \\ School of Physics and Astronomy. University of Minnesota. Minneapolis, Minnesota 55455
}

This department in collaboration with the Committee on Apparatus of the AAPT will welcome the submission of brief communications reporting new equipment, techniques, or materials of interest to teachers of physics. Notes on new applications of older apparatus, measurements supplementing data supplied by manufacturers, information which, while not new, is not generally known, procurement information, and news about apparatus under development are suitable for publication in this section. Neither the American Journal of Physics nor the Editors assume responsibility for the correctness of the information presented. Submit materials to: Bruce G. Eaton, Department of Physics, University of Minnesota, 116 Church St. SE, Minneapolis, MN 55455.

\section{A sensitive photodiode photometer for laboratory experiments}

\author{
David L. DuPuy \\ Department of Astronomy, Saint Mary's University, Halifax, Nova Scotia B3H 3C3, Canada
}

Many laboratory experiments require a light detector, and the venerable photomultiplier tube is often more clumsy and expensive than is desirable for undergraduate labs. Likewise, many undergraduates are more inclined to tinker with a low-voltage, solid-state photometer than with highvoltage supplies, etc. This paper describes a practical circuit for an inexpensive photometer which employs a PIN photodiode and a simple, inexpensive, high-gain op-amp circuit. The photometer is remarkably sensitive, easily reading the regular changes in opacity through a fingertip as blood is pumped through the finger.

The circuit is shown in Fig. 1. The op-amp is the ubiquitous 741 , available for as little as $25 \notin$. Pin connections in Fig. 1 are shown for the 8-pin mini-DIP package; pin connections vary for other packages. The PIN-125 photodiode from United Detector Technology ${ }^{1}$ (UDT) represents my choice of photodiode, considering cost, sensitivity, and availability. The spectral response is broad, peaking around $8500 \AA$, with a half-power bandwidth of $6000 \AA$. The cost of this device is $\$ 7$.

The circuit shown is a relatively standard current-tovoltage converter, and the gain is approximately ( $2 \mathrm{~V} /$ $\mu \mathrm{A})\left(R_{2} / R_{1}\right)$. Resistors $R_{1}$ and $R_{2}$ make up a gain-multi-

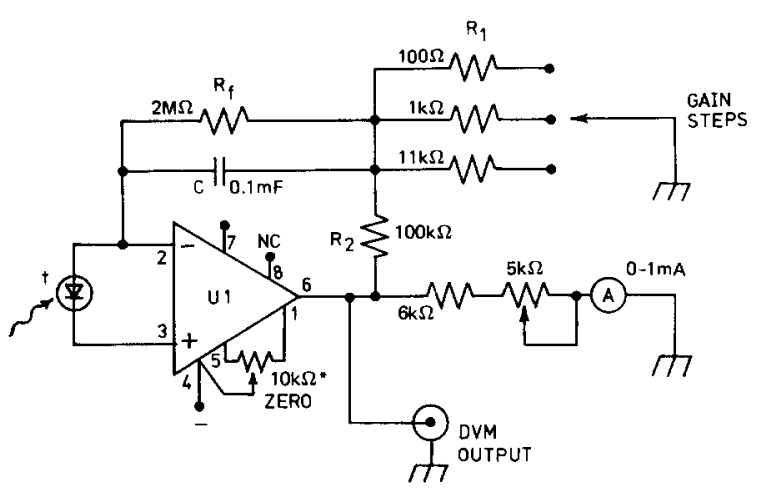

\footnotetext{
* zero set; miniature multiturn potentiometer

† UDT PIN-125 PHOTODIODE

U 741 OP-AMP for RCA CA 3140\}
}

Fig. 1. Circuit diagram for the photodiode photometer. See text for details. plication circuit, which effectively increases the resistance of the feedback circuit. The total gain is thereby increased without physically increasing the value of the feedback resistor $R_{f}$. That is an important consideration, since resistors larger than about $10 \mathrm{M} \Omega$ are expensive, or noisy, or both. For the circuit shown, the largest practical gain results for $R=100 \Omega$. An inexpensive FET input op-amp (e.g., the RCA CA3140, about $80 \phi$ ) may be used for measurement of even lower light levels. With the panel meter shown, adjustment can be made for saturation just off scale, to avoid false readings being obtained if saturation in the 741 occurred at, say, $90 \%$ scale deflection. This may also help avoid damage to the meter from exposure to unexpectedly high light levels. The photodiode current may be estimated in this circuit by $i_{p}=E_{\mathrm{out}} / R_{\mathrm{eff}}$, where $R_{\mathrm{eff}}$ is approximately $R_{f}\left(R_{2} / R_{1}\right)$.

The cost of the photometers built here ranged from about $\$ 20$ (including meter and minibox) for the battery versions with a panel meter readout, to about $\$ 70$ for a classroom demonstration version with large digital LED readout. The gain steps shown are one decade steps, and these gain steps should be calibrated before combining readings from more than one range. The time constant is determined by the value of $R_{f} C$, and precautions should be taken to ensure that the time constant is smaller than the recording interval.

The PIN-125 photodiode is operated in the photovoltaic mode, with no reverse bias applied. Some photodiodes are designed to be operated in the photoconductive mode, with a reverse bias of a few volts. Some (e.g., the UDT PIN-3) may be used equally well in either mode. The advantages of the photoconductive mode include extremely linear response with light level, and very fast response. The advantages of the photovoltaic mode are simplicity and no dark current. Since use of the photometer at low light levels was of concern in our application, the photovoltaic mode was chosen. For measurement of photodiode currents below about $0.1 \mu \mathrm{A}$, the 741 is no longer suitable, and an op-amp with a much lower input bias current must be chosen. The RCA CA3140 is a good choice (10-pA typical input bias current).

The photovoltaic mode offers sufficient linearity for most applications, and an attempt to measure the photodiode response to different light levels makes a good laboratory 


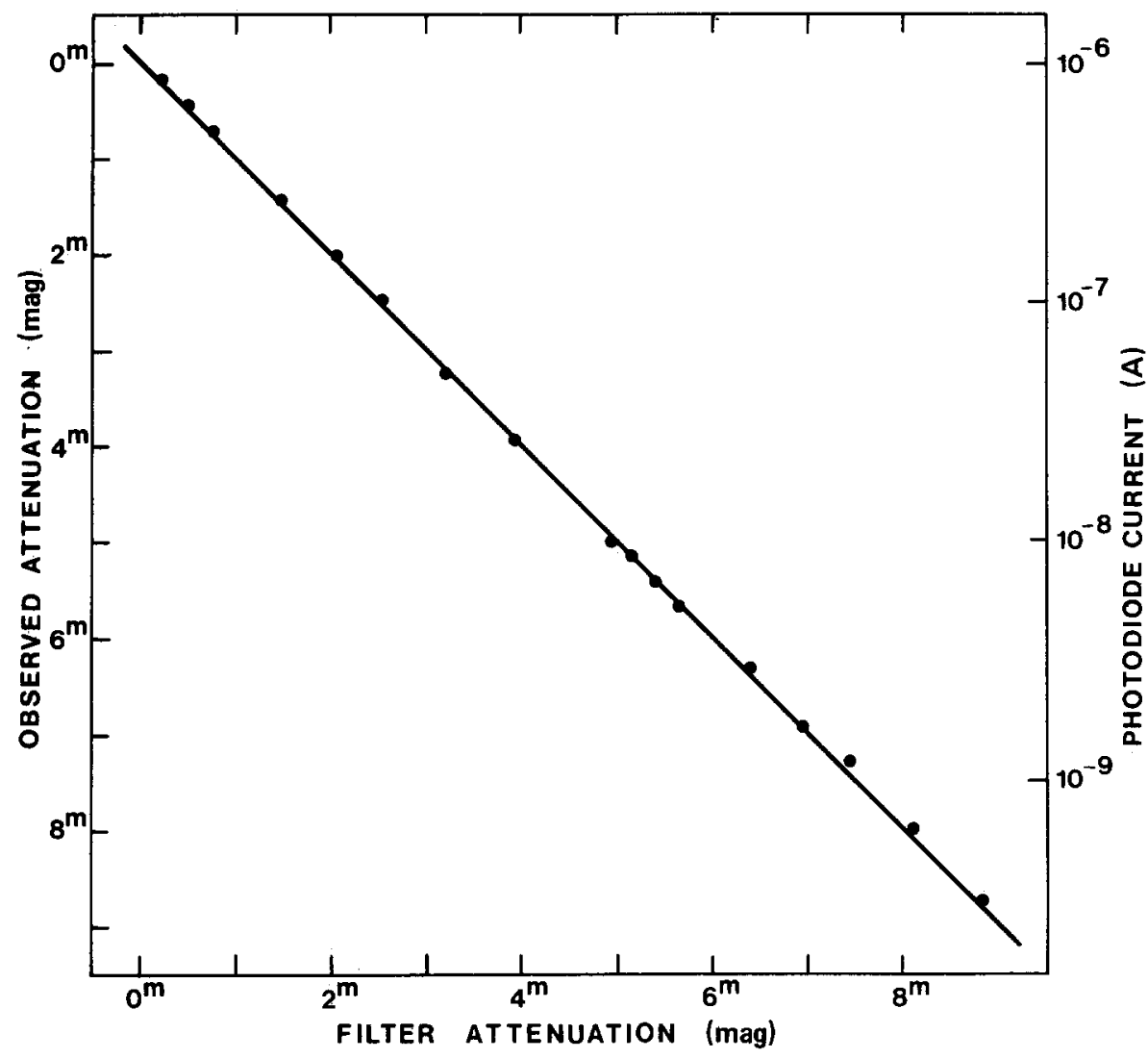

Fig. 2. Results from a simple experiment to measure the photometer response to different light levels. The filter attenuation is plotted in magnitudes on the abscissa, and the observed photometer output is plotted in magnitudes on the ordinate [mag $=$ const $-2.5 \log _{10}$ (voltmeter reading)]. The photodiode current is plotted on the right-hand side ordinate, in $\mathbf{A}$.

experiment for advanced students. Results are shown in Fig. 2 from a simple experiment to measure photometer response, using the circuit shown in Fig. 1 (with a CA3140) with changes in light level over a range of almost $10^{4}$. The straight line is drawn with unit slope. Balzers thin-film neutral-density filters were employed to attenuate the light beam by known amounts. The uncertainty in the filter attenuation contributes appreciably to the measuring uncertainty. For example, the transmittance of the $1 \%$ neutral-density filter used here was measured independently to be $0.0109 \pm 0.0002$. A far better approach to controlling filter attenuation is to employ one of the superbly linear transmittance standards made by Technometrics, Inc. ${ }^{2}$ To avoid possible red leak, an interference filter at $6529 \AA$ was inserted in the beam (the photodiode is sensitive out to about $1 \mu \mathrm{m})$. Stray light inside the apparatus enclosure became a problem at the lower light levels, and some improvement resulted after several light baffles were installed. Finally, a problem arose from an unexpected direction: from behind the photodiode! The insulating material around the active lead on the rear of the case was translucent and even the reduced room light produced undesirable effects. The slight variations from linear response in Fig. 2 around $10^{-9} \mathrm{~A}$ are probably caused by stray light inside the relatively crude experimental enclosure; more precise deductions about any intrinsic nonlinearities cannot be made until the precision of this experiment is improved. Nonetheless, it is hoped that the problems and results elaborated here will be helpful to others who wish to employ a photodiode photometer for teaching and lab experiments. The photometers here have been used primarily with a digitally synthesized variable star in astronomy classes; details are given elsewhere. ${ }^{3}$

\footnotetext{
${ }^{1}$ United Detector Technology, 2644 30th St., Santa Monica, CA 90405.

${ }^{2}$ Technometrics, Inc., 1220 Potter Dr., West Lafayette, IN 47906.

${ }^{3}$ D. L. DuPuy, J. R. Astron. Soc. Can. 71, 199 (1977).
}

\title{
Demonstration of transient beats
}

\author{
H. S. T. Driver \\ Physics Department, University of Cape Town, Rondebosch 7700, South Africa
}

The harmonic oscillator occupies a position of central importance in physics and students should be encouraged to develop some physical insight into the behavior of harmonic oscillators in addition to obtaining a grasp of the mathematics of harmonic motion. This insight can probably best be acquired by doing experiments, a wide range of these being available even to modestly equipped laboratories. Few of these experiments, however, demonstrate the interaction between the initial transient and the steady-state motion of a harmonically driven oscillator. In this Note it is shown how an operational amplifier circuit which simulates a harmonic oscillator can be used to demonstrate this interaction; the circuit is also capable of demonstrating many other aspects of the behavior of free and driven oscilla- 